\title{
Effects of breast density and compression on normal breast tissue hemodynamics through breast tomosynthesis guided near-infrared spectral tomography
}

Kelly E. Michaelsen

Venkataramanan Krishnaswamy

Linxi Shi

Srinivasan Vedantham

Andrew Karellas

Brian W. Pogue

Keith D. Paulsen

Steven P. Poplack 


\title{
Effects of breast density and compression on normal breast tissue hemodynamics through breast tomosynthesis guided near-infrared spectral tomography
}

\author{
Kelly E. Michaelsen, ${ }^{a, *}$ Venkataramanan Krishnaswamy, ${ }^{a}$ Linxi Shi, ${ }^{b}$ Srinivasan Vedantham, ${ }^{c}$ Andrew Karellas, ${ }^{c}$ \\ Brian W. Pogue, ${ }^{a}$ Keith D. Paulsen, ${ }^{a}$ and Steven P. Poplack ${ }^{d}$ \\ aDartmouth College, Thayer School of Engineering, 14 Engineering Drive, Hanover, New Hampshire 03755, United States \\ ${ }^{\mathrm{b}}$ Georgia Institute of Technology, School of Mechanical Engineering, 801 Ferst Drive, Atlanta, Georgia 30332, United States \\ 'University of Massachusetts Medical School, Department of Radiology, 55 Lake Avenue North, Worcester, Massachusetts 01655, United States \\ 'Washington University School of Medicine, Mallinckrodt Institute of Radiology, 4921 Parkview Place, St. Louis, Missouri 63110, United States
}

\begin{abstract}
Optically derived tissue properties across a range of breast densities and the effects of breast compression on estimates of hemoglobin, oxygen metabolism, and water and lipid concentrations were obtained from a coregistered imaging system that integrates near-infrared spectral tomography (NIRST) with digital breast tomosynthesis (DBT). Image data were analyzed from 27 women who underwent four IRB approved NIRST/DBT exams that included fully and mildly compressed breast acquisitions in two projections - craniocaudal (CC) and mediolateral-oblique (MLO) - and generated four data sets per patient (full and moderate compression in CC and MLO views). Breast density was correlated with $\mathrm{HbT}(r=0.64, p=0.001)$, water $(r=0.62, p=0.003)$, and lipid concentrations $(r=-0.74, p<0.001)$, but not oxygen saturation. CC and MLO views were correlated for individual subjects and demonstrated no statistically significant differences in grouped analysis. Comparison of compressed and uncompressed imaging demonstrated a significant decrease in oxygen saturation under compression $(58 \%$ versus $50 \%, p=0.04)$. Mammographic breast density categorization was correlated with measured optically derived properties. @ 2016 Society of Photo-Optical Instrumentation Engineers (SPIE) [DOI: 10.1117/1.JBO.21.9.091316]
\end{abstract}

Keywords: near-infrared; mammography; breast tomosynthesis; compression.

Paper 160140SSRR received Mar. 6, 2016; accepted for publication Aug. 30, 2016; published online Sep. 27, 2016.

\section{Introduction}

Despite proven benefit in breast cancer specific mortality, ${ }^{1,2}$ the utility of screening mammography has been called into question on the basis of limited specificity ${ }^{3,4}$ and overdiagnosis. ${ }^{5,6}$ Digital breast tomosynthesis (DBT) has shown potential to mitigate these limitations based on reduction in screening recall rate and preferential detection of invasive carcinoma. ${ }^{7,8}$ The U.S. Preventive Services Task Force in its most recent recommendation concluded that there was insufficient evidence to make a recommendation for DBT as a primary screening tool. ${ }^{4}$ There is a need for a physiology-based screening tool that is not constrained by dense breast composition, and one that may identify breast cancer that is more likely to cause morbidity and mortality over a subject's lifetime.

Physiology-based imaging, such as near-infrared spectral tomography (NIRST), is an ideal adjunct. It does not require administered contrast agents, making it suitable for imaging almost all women. NIRST uses light wavelengths between 650 and $1000 \mathrm{~nm}$ to image several functional markers in tissue, including hemoglobin concentration, oxygen saturation, and water and lipid levels. When used by itself, NIRST suffers from poor spatial resolution due to pervasive light scattering. However, NIRST has demonstrated improved resolution to nearly $1 \mathrm{~mm}^{9}$ when paired with other modalities, including $\mathrm{x}$-rays, ultrasound, and magnetic resonance imaging (MRI).

*Address all correspondence to: Kelly E. Michaelsen, E-mail: Kelly.e. michaelsen@dartmouth.edu
Earlier work by Fang et al. ${ }^{10}$ demonstrated the potential for NIRST combined with DBT to distinguish benign from malignant lesions in a trial of over 100 women. The study found higher total hemoglobin and higher scattering coefficients in cancer compared to benign lesions and fibroglandular tissue within the same breast. ${ }^{10}$

We have developed a prototype NIRST/DBT system that expands on the approach described by Fang et al. by (i) exploiting DBT data to derive anatomical priors to improve NIRST image resolution and accuracy of the recovered optical properties $^{11}$ and (ii) adding more optical illumination wavelengths (8 versus 2) and light detectors (75 versus 40). ${ }^{12}$ These advancements enable assessment of water and lipid content, in addition to hemoglobin and oxygen saturation. One challenge with NIRST/DBT is the use of breast compression, which is required only for the DBT part and can alter these physiological signatures, notably the hemoglobin and oxygenation status of breast tissue in both normal and malignant cases. ${ }^{13}$

In this study, the largest cohort of women with normal (BIRADS 1) mammography was imaged with the coregistered NIRST/DBT under two compression levels to evaluate optically derived tissue property changes. Subjects selected for this study included a range of breast densities enriched with a larger proportion of women with dense breasts, a subgroup that is less well served by mammography and at greater risk of developing breast cancer. ${ }^{7}$ The purpose of this study was to benchmark 
optically derived tissue properties across a range of breast densities and assess the effects of compression on estimates of hemoglobin, oxygen metabolism, and water and lipid concentrations obtained from the coregistered NIRST/DBT imaging system.

\section{Materials and Methods}

Subjects enrolled in the study followed experimental protocols that were approved by our Institutional Review Board. Written informed consent was obtained from all participants. The study was compliant with Health Insurance Portability and Accountability Act guidelines. Sequential eligible subjects were selected for enrollment to provide a range of breast size and mammographic breast density that was enriched with greater breast density, i.e., more subjects with heterogeneous or extremely dense breasts than scattered or predominantly fatty breasts. Selection was based on the clinical radiology report of breast density and subject reported bra cup size. Subjects were imaged with NIRST/DBT from June 2013 to March 2014.

\subsection{Imaging Instrumentation}

The NIRST/DBT imaging system is a modified Hologic ${ }^{\odot}$ research DBT prototype ${ }^{14}$ augmented with NIRST. ${ }^{12}$ The optical components were integrated into the DBT platform for image coregistration. The light source was mounted to the DBT gantry and did not interfere with DBT acquisition, whereas an optical detector panel slides into a polycarbonate casing and is removed during X-ray exposure. ${ }^{15}$

The DBT system was configured for 11 acquisitions recorded over a span of $26 \mathrm{deg}$. The reconstructed images had resolutions of $0.125 \mathrm{~mm}(125 \mu \mathrm{m})$ in orthogonal ( $x$ and $y$ ) planes and $1 \mathrm{~mm}(1000 \mu \mathrm{m})$ in the depth $(z)$ direction. For NIRST, laser diodes of eight wavelengths (660, 785, $808,830,852,905,915$, and $940 \mathrm{~nm}$ ) were scanned across the top of the breast and illuminated the surface at 77 positions. These 77 positions formed a grid of $11 \times 7$ points spaced $1,1.5$, or $2 \mathrm{~cm}$ apart depending on breast size. Light was collected on a detector panel comprised of 75 photodiodes placed beneath the breast in an 11 ( 9 in the row furthest from the patient) $\times 7$ grid with $2 \mathrm{~cm}$ spacing between the detector centers. When the $2 \mathrm{~cm}$ illumination spacing was used, the source and detectors were aligned on either side of the breast. In other cases, the source grid was shifted toward the chest wall and middle of the breast to maximize usable data from smaller breast surfaces.

\subsection{Examination Protocols}

Each subject had NIRST exams in mild and full compression and a DBT exam in full compression. Mild compression was intended to prevent breast motion during breathing and was based on the technologist's and patient's assessment that the breast was adequately secured in place. Full compression was intended to maximally compress the breast without causing pain and was based on usual clinical practice. This practice typically led to a 1 to $2 \mathrm{~cm}$ decrease in breast thickness from the mildly compressed to fully compressed states.

For each projection, the imaging protocol was sequenced as: (i) NIRST with mild compression, (ii) NIRST with full compression, and (iii) DBT. In detail, the optical detector was placed underneath a polycarbonate detector casing prior to examination and images of a calibration phantom were obtained. The breast was positioned by a technologist with 45 years of clinical mammography experience. After breast positioning and light blocking drapes were placed, the first NIRST acquistion (45 s) was performed with the breast in mild compression. Full compression was then applied by the technologist without altering the participant's position, and the second NIRST exam was acquired (40 s). The optical detector was removed and the DBT exam was performed (12 s). The process was repeated in the mediolateraloblique (MLO) geometry or in the contralateral breast. The time from subject arrival to exit was approximately $25 \mathrm{~min}$.

The first 15 subjects were also imaged with a separate standalone diffuse optical spectroscopic imaging (DOSI) system. ${ }^{16}$ For the DOSI exam, the subject sat or reclined in a chair while the reflectance probe was gently placed on the breast surface. Twenty recordings were obtained: four measurements were taken in a square pattern in each quadrant and four interquadrant measurements were acquired. The process required about $10 \mathrm{~min}$ and all data points were analyzed and averaged to obtain a bulk tissue scattering estimate that was available for NIRST/ DBT image reconstruction.

\subsection{Data Postprocessing}

The 11 DBT projection images were reconstructed using the filtered backprojection method and formatted into a threedimensional image stack and segmented into adipose and fibroglandular tissues using an algorithm that employs bilateral filtering to reduce out-of-plane artifacts. ${ }^{17,18}$ These segmented images created a region-tagged mesh that incorporated anatomic information from the DBT data and were used to reconstruct the NIRST results.

NIRST data were calibrated with homogeneous optical phantom measurements. Data points outside the breast volume were removed, including those falling within $15 \mathrm{~mm}$ of the breast boundary, outside the linear range of the optical detector, or more than two standard deviations from the best fit line of the log of amplitude versus source-optical detector separation distance.

\subsection{Image Reconstruction}

NIRST image reconstructions were spectrally and spatially constrained. In this approach, a single value was assigned to the adipose region and another was associated with the fibroglandular region, significantly reducing the number of unknown variables in the image reconstruction process and improving accuracy in the recovered tissue optical properties. ${ }^{19}$ The NIRFAST software package was used to recover hemoglobin concentration, oxygen saturation, and water and lipid content of the breast based on a finite element image reconstruction technique. ${ }^{20}$ Scattering was measured with the DOSI system for a subgroup of 15 women, and the mean value was applied to NIRST image reconstructions of all subjects in the study.

\subsection{Statistical Analysis}

Student $t$ tests were used to assess differences between the demographic groups shown in Table 2 including breast density, fibroglandular content, bra cup size, breast thickness, body mass index (BMI), and DOSI. Average breast property values were determined from the ratio of fibroglandular and fat tissue to total breast volume in order to include the effects of the two tissue types proportionally. Paired $t$-testing was performed between each of the different subsets. For continuous data 

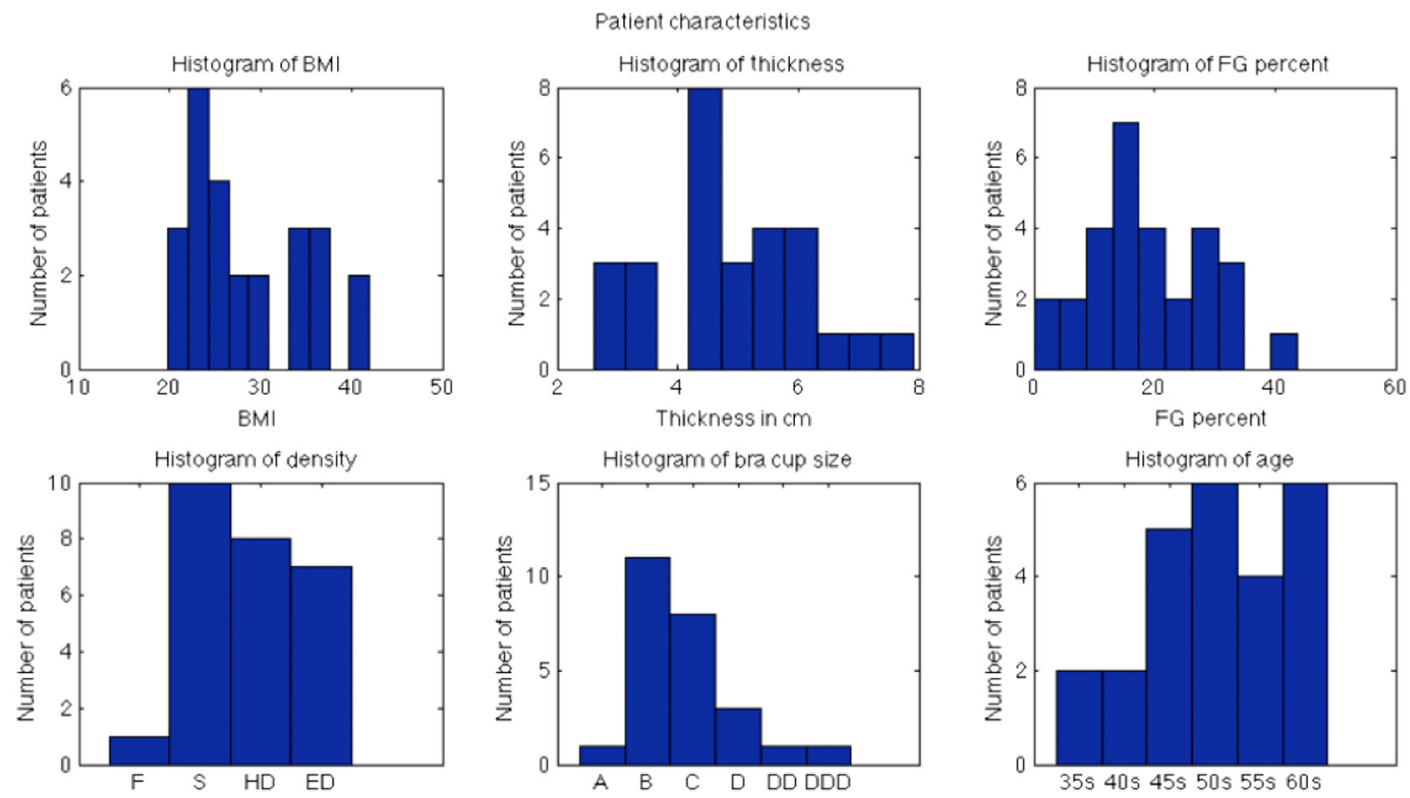

Fig. 1 Histograms of key demographic and clinical information for the subjects enrolled in the study.

comparisons, correlation and $p$ values were determined to assess statistical significance of variables. All statistical assessments were performed using the MATLAB software package and $p$ values $<0.05$ were considered significant.

\section{Results}

Thirty-two women with normal mammography (BI-RADS 1) within the prior 2 years were enrolled and underwent the NIRST DBT imaging protocol. The first five subjects were excluded due to poor DBT quality and unrecorded laser power settings that could not be determined retrospectively. Of the remaining 27 subjects, the data of one woman were restricted to the craniocaudal (CC) projection due to a software malfunction prior to the MLO acquisition. This yielded four image sets per subject (CC fully compressed, CC mildly compressed, MLO fully compressed, MLO mildly compressed), except for the one subject who was imaged only in the $\mathrm{CC}$ view; in that case, there were only two sets of images. In total $26 \times 4+1 \times 2$ images were obtained, half mild and half fully compressed. The exact numbers include 53 NIRST mildly compressed, 53 NIRST fully compressed, and 53 DBT fully compressed image acquisitions.

Figure 1 summarizes the characteristics of the women imaged, including their BMI, breast thickness, percent fibroglandular tissue, ${ }^{18}$ breast density, bra cup size, and age. The average BMI was 28.5, nearly 29-the US average for older women. $^{21}$ The average age was 51 years. The average compressed breast thickness was $4.9 \mathrm{~cm}$, similar to other studies (4.4 to $5.4 \mathrm{~cm}) .^{22,23}$ The average fibroglandular content of $19 \%$ was marginally higher than the average population $(10 \%$ to $17 \%)^{24,25}$

\subsection{Optically Derived Breast Tissue Properties}

Mean $\mathrm{HbT}$ was $14.3 \pm 6.4 \mu \mathrm{M}$, oxygen saturation was $53 \pm 17 \%$, water fraction was $12 \pm 9 \%$, and lipid content was $34 \pm 18 \%$ for all exams (including both full and mild compression), as shown in Table 1 with confidence intervals. Additionally, examination of adipose and fibroglandular tissues separately showed mean $\mathrm{HbT}$ of 14.1 versus $15.1 \mu \mathrm{m}$, oxygen saturation of $55 \%$ versus $48 \%$, water content of $11 \%$ versus $14 \%$, and lipid content of $35 \%$ versus $29 \%$, respectively, but none of these differences was statistically significant. This is not surprising, given the interpatient variability of breast tissue. For the group imaged with both the DBT and DOSI systems, a comparison of the optically derived property estimates using the individually recorded scattering versus the group averaged scattering demonstrated minimal differences ( $r$ values for oxy-, deoxy-hemoglobin, and water were all greater than 0.9 , data not shown). Comparing CC and MLO breast positions
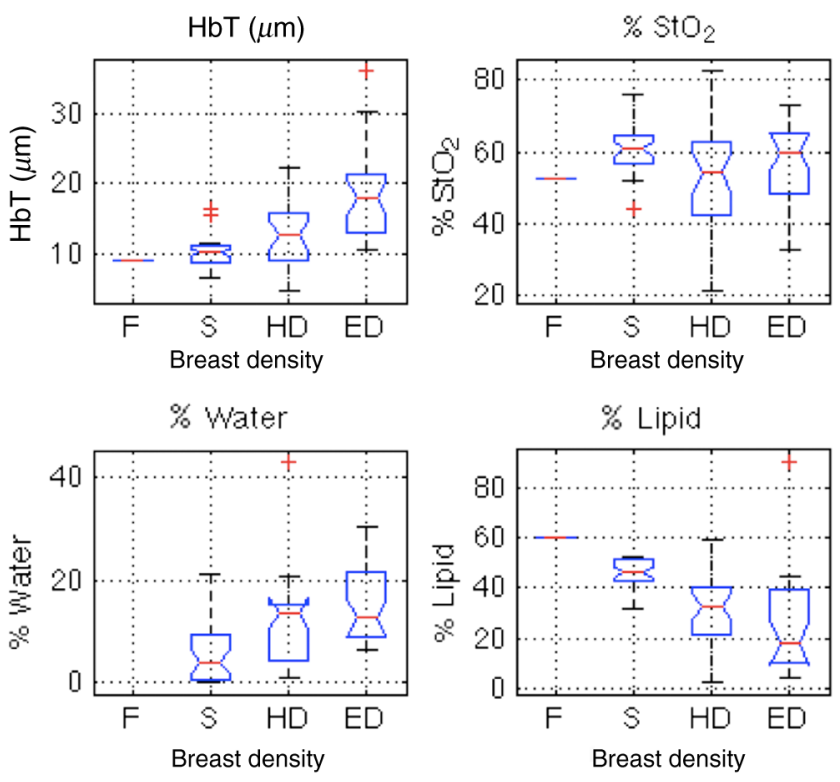

Fig. 2 Comparison of optical data for total hemoglobin, oxygen saturation, and water and lipid concentrations for different breast density categories ( $F$, fatty; S, scattered; HD, heterogeneously dense; ED, extremely dense). Statistical significance between breast density categories occurs where angled portions of the box plot bands do not overlap between two bars. 
Table 1 Review of selected publications of optically derived tissue properties of normal breast relative to the current study. Average values are shown with standard deviations in parentheses.

\begin{tabular}{|c|c|c|c|c|c|}
\hline Study source & Number of subjects & Total $\mathrm{Hb}(\mu M)$ & $\mathrm{O}_{2}$ Sat $\%$ & Water \% & Lipids \% \\
\hline Dartmouth NIRST/DBT & 27 & $14.3(6.4)$ & $53(17)$ & $12(9)$ & $34(18)$ \\
\hline $\begin{array}{l}\text { Ref. } 26 \text { (pre- and postmenopausal } \\
\text { subjects) }\end{array}$ & 95,107 & $15.7(4.9), 12.4(4.7)$ & $70.5(7.6), 68.5(8)$ & $21.3(7.2), 18.2(5.9)$ & $\begin{array}{l}61.5(10.2) \\
68.3(9)\end{array}$ \\
\hline Ref. 27 & 49 & $20.4(7.1)$ & $71.1(7.7)$ & $28.9(11.7)$ & $62.4(12.6)$ \\
\hline Ref. 28 & 60 & $16.6(4.6)$ & $68.3(7.2)$ & $51.5(14.1)$ & \\
\hline $\begin{array}{l}\text { Ref. } 29 \text { (pre- and postmenopausal } \\
\text { subjects) }\end{array}$ & 93,102 & 13.64(4.1), 10.4(3.2) & 86.8(9.7), 84.9(9.1) & & \\
\hline Ref. 10 & 138 & $19.2(6.5)$ & $73(6)$ & & \\
\hline Ref. 30 & 87 & $17.3(6.3)$ & $74(7)$ & & \\
\hline Ref. 31 & 68 & $16.2(6.1)$ & 71 & & \\
\hline Ref. 32 & 58 & $17.5(7.5)$ & $67.7(9.3)$ & & \\
\hline Ref. 33 & 14 & $18(5)$ & $69(6)$ & & \\
\hline Ref. 34 & 27 & $12(6)$ & $71(10)$ & & \\
\hline Ref. 35 & 3 & $17(2)$ & $68(4)$ & & \\
\hline Ref. 36 & 12 & $25.3(8.3)$ & $70(7)$ & & \\
\hline Ref. 37 & 19 & $22.3(7.5)$ & $64.8(10.3)$ & & \\
\hline Ref. 38 & 55 & $21.2(11.7)$ & & & \\
\hline Ref. 39 & 15 & $21.8(9.2)$ & & & \\
\hline
\end{tabular}

demonstrated no significant differences between the views as a whole (data not shown). In comparing individual subjects, statistically significant correlations in hemoglobin, oxygen saturation, and lipids between the two views were confirmed ( $r=0.6$, $0.6,0.7$, respectively). Mean water values were within $1 \%$ when comparing CC and MLO and were not significantly different between the two groups, but when examining the correlation of CC and MLO views for individual women it did not reach a level of statistical significance $(p=0.14)$ as there was less variation in water values across women than there was in lipid content.

As shown in Fig. 2, significant correlations between optically derived tissue properties and breast density were observed. The difference in $\mathrm{HbT}$ and lipids was statistically significant among the three higher density groups. No statistically significant differences in oxygen saturation occurred across the groups. Average values are compared to data from previous reports in the literature in Table 1. A full list of correlations between optically derived tissue properties and clinical parameters is summarized in Table 2.

Fibroglandular content was statistically correlated with total hemoglobin, water, and lipids, and opposite to the correlation observed in BMI (data not shown). The only significant correlation between bra cup size and optically derived tissue properties was found with oxygen saturation as shown in Fig. 3 and listed in Table 2. Although trends are the same for both bra cup size and thickness, bra cup size did not demonstrate significant correlation with lipid and water content, likely reflecting the self-reported nature of this data point.

\subsection{Correlation with Compression}

As shown in Fig. 4, optically derived properties were similar in fully compressed and mildly compressed cases with no significant differences except for oxygen saturation $(p=0.04)$-an average of $58 \%$ for the mildly compressed relative to $50 \%$ for the fully compressed cases. Additionally, breast thickness was highly correlated with oxygen saturation as well as with water and lipid content as seen in Table 2.

\section{Discussion}

Mean $\mathrm{HbT}$ was $14.3 \mu \mathrm{M}$, which is on the low end of the previously reported values shown in Table 1 , but is similar to the report by Fang et al. $\left(16 \mu \mathrm{M}^{31}\right.$ and $\left.19 \mu \mathrm{M}^{10}\right)$. Both water and lipid contents were also lower than previously published results as shown in Table 1. This finding is likely influenced by light channeling in the plastic compression paddles, as the effect was similar for every participant, all of whom were examined with the same compression paddles. ${ }^{15}$ The effect is more significant for water and lipids than for hemoglobin content because light channeling increases at longer wavelengths, coinciding with the higher absorption spectra for water and lipids. Additionally, lower lipid content would be expected since the fatty breast subject population was underrepresented. 
Table 2 Correlations between breast density, bra cup size, BMI, fibroglandular volume fraction, breast thickness, and DOSI with DBT/NIRST optically derived tissue properties using Student $t$ tests. Bolded values indicate statistical significance.

\begin{tabular}{|c|c|c|c|c|c|c|c|c|}
\hline Breast density & Correlation & $p$ value & Bra cup size & Correlation & $p$ value & $\mathrm{BMI}$ & Correlation & $p$ value \\
\hline $\mathrm{HbT}$ & 0.64 & 0.001 & $\mathrm{HbT}$ & -0.18 & 0.42 & $\mathrm{HbT}$ & -0.45 & 0.034 \\
\hline $\mathrm{StO}_{2}$ & -0.10 & 0.66 & $\mathrm{O}_{2}$ Sat & 0.54 & 0.01 & $\mathrm{O}_{2}$ Sat & 0.25 & 0.27 \\
\hline $\mathrm{H}_{2} \mathrm{O}$ & 0.62 & 0.003 & $\mathrm{H}_{2} \mathrm{O}$ & -0.28 & 0.22 & $\mathrm{H}_{2} \mathrm{O}$ & -0.38 & 0.09 \\
\hline Lipids & -0.74 & $<0.001$ & Lipids & 0.27 & 0.24 & Lipids & 0.646 & 0.002 \\
\hline Fibroglandular content & Correlation & $p$ value & Thickness & Correlation & $p$ value & DOSI & Correlation & $p$ value \\
\hline $\mathrm{HbT}$ & 0.68 & $<0.001$ & $\mathrm{HbT}$ & -0.18 & 0.39 & $\mathrm{HbT}$ & 0.47 & 0.30 \\
\hline $\mathrm{StO}_{2}$ & -0.12 & 0.58 & $\mathrm{O}_{2}$ Sat & 0.71 & $<0.001$ & $\mathrm{O}_{2}$ Sat & 0.15 & 0.75 \\
\hline $\mathrm{H}_{2} \mathrm{O}$ & 0.63 & 0.001 & $\mathrm{H}_{2} \mathrm{O}$ & -0.63 & 0.001 & $\mathrm{H}_{2} \mathrm{O}$ & 0.73 & 0.07 \\
\hline Lipids & -0.80 & $<0.001$ & Lipids & 0.56 & 0.005 & Lipids & 0.92 & 0.003 \\
\hline
\end{tabular}

A more specific and individualized assessment of the NIRST/ DBT system was obtained by comparing data from 15 women who were imaged with both DOSI and NIRST/DBT. DOSI has demonstrated successful differentiation of benign and malignant tissues, the ultimate goal of NIRST/DBT imaging. ${ }^{32}$ This comparison was important for two reasons. First, optically derived tissue properties from the two systems should be similar in these patients, and the DOSI data were useful in verifying results from the new NIRST/DBT system, although identical values are not expected given differences in imaging geometry and the volume of tissue assessed by the two approaches. Second, scattering values cannot be estimated from our NIRST/DBT continuous wave acquisitions and availability of DOSI values was important for assessing the consequences of not obtaining subject-specific optical scattering estimates for NIRST data/image analysis of the remaining participants. The impact of using individually measured DOSI scattering properties versus the average scattering value from the whole group on other optically derived
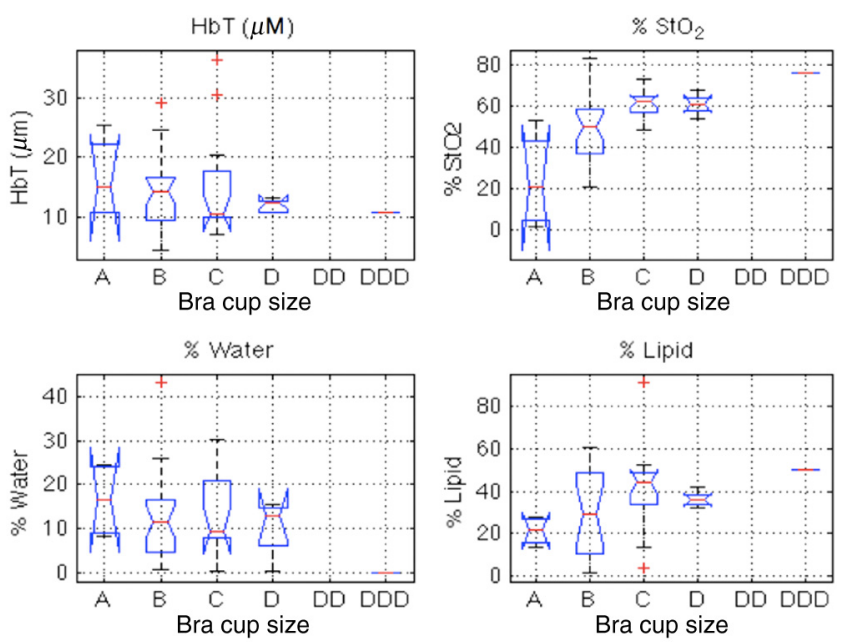

Fig. 3 Comparison of total hemoglobin, oxygen saturation, and water and lipid concentrations with subject reported bra cup size. Statistical significance between categories occurs where angled portions of the bands do not overlap. tissue properties was relatively modest ( $r>0.9$ for oxy- and deoxy-hemoglobin and water, data not shown). Hence, the DOSI-derived averaged scattering may be used in lieu of breast-specific scattering data with minimal effects on the resulting breast-specific absorption values. Although the data from breasts imaged with both DOSI and NIRST/DBT systems demonstrated a small 3\% difference in water content (15\% for DOSI and $12 \%$ for NIRST/DBT), but a larger disparity in lipid fraction (34\% versus $58 \%$ for NIRST/DBT versus DOSI), these differences are likely due to the preferential subcutaneous fat sampling of DOSI compared to NIRST/DBT, which probes the entire breast volume- - a hypothesis further supported by the correlation between the fat values found in NIRST/DBT and DOSI $(r=0.92, p=0.003)$.

In comparing results from the CC and MLO views, no statistically significant difference was found between the two groups as a whole, a reassuring result for reproducibility. Significant correlations in hemoglobin, oxygen saturation, and lipids existed between the two views for individual subjects, supporting the potential of using continuous wave near-infrared
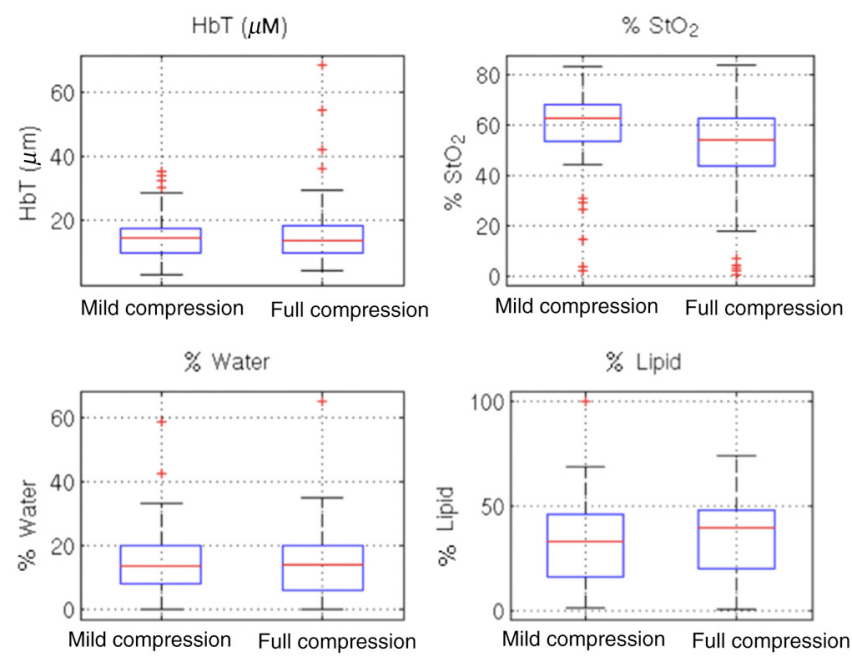

Fig. 4 Comparison of optically derived tissue property estimates in mildly compressed and fully compressed views. 
methods for clinical breast imaging — a less expensive, less complex and thereby more easily integrated approach into the clinic, which requires efficient workflow. Furthermore, the rapidity of the NIRST acquisition, which is a much faster alternative to frequency or time domain optical methods, allows seamless integration into the clinical examination.

Our results confirm expected relationships between optically derived tissue properties and subject characteristics including breast density, fibroglandular volume, and BMI. While these correlations have been noted for stand-alone optical imaging systems, heretofore, they have not been demonstrated in optical fusion technology based on spatial priors from DBT and continuous wave optical data acquisition in the compressed breast. Denser breasts and breasts with higher fibroglandular volume demonstrated significantly higher water and hemoglobin content and lower lipid levels without demonstrable correlation with oxygen saturation. Increasing BMI showed opposite trends, with higher recovered lipid fractions and lower hemoglobin concentrations.

The degree of breast compression did not significantly alter total hemoglobin content as in prior reports. Previous studies involved imaging systems in which light sources and detectors were on the same side of the breast, limiting the sampling volume and preferentially probing more superficial tissues. Blood may be redistributed toward the stiffer, deeper fibroglandular tissue that can be detected by NIRST/DBT. Only one prior study by Busch et al. used transmission measurements of the breast under compression, and demonstrated changes in hemoglobin and oxygenation, as well as an $88 \%$ decrease in blood flow at mammographic compression levels. ${ }^{40}$ Although a trend toward lower hemoglobin was noted in our study, the result was not statistically significant, and may have been due to unaccounted changes in optical scattering.

In contrast, full breast compression had a substantial effect on tissue oxygenation with a significant decrease occurring under full compression $(p=0.04)$. Reduction in oxygen saturation is expected during compression, assuming blood flow is restricted, since tissues consume the remaining oxygen in the static blood to meet ongoing metabolic demands. Prior studies with fewer subjects have demonstrated similar results. ${ }^{41,42}$ Additionally, oxygen saturation was lower in this study when compared to literature values, and may be due to compressive effects that occur even under mild compression, which would not be present in previous data as most other systems do not place any pressure on the breast tissue during examination.

A simpler approach to some of the analyses presented here would be to ignore spatial information and analyze the whole breast as homogeneous. However, the next step for this imaging system is to examine women with breast lesions, particularly the small lesions found on breast screening exams. Homogeneous analysis would likely be incapable of detecting minute changes in optical properties expected in small tumors. Spatial priors have been shown to improve quantification of tissue properties and incorporating this information simulates future abnormal breast studies more closely than a homogeneous analysis. ${ }^{11}$

Several limitations in the study are related to subject selection, system design, and compression measurements. The sample of subjects is not representative of the general mammographic screening population, as we intentionally chose women with denser breasts and recruited only a single subject with fatty composition. The continuous wave signals acquired with NIRST do not measure differences in scattering, an optical property that has been shown to differ in benign and malignant tissue. ${ }^{10}$ Unfortunately, our research NIRST/DBT system was not equipped with a compression force sensor, so breast thickness was used as surrogate. In terms of the lipid and water changes, the results reported here may be confounded by other variables such as BMI, which was highly correlated with breast thickness $(r=0.79, p<0.001)$, and shows the same trends as seen for thickness in regards to water and lipids. However, BMI was not related to changes in oxygen saturation, and a significant correlation was found between breast thickness and oxygen saturation $(r=0.71, p<0.001)$, which can be attributed to the compressive forces applied to the breast.

In summary, our results benchmark optically derived tissue properties estimated through the NIRST/DBT system and reveal some important differences between our completely coregistered data and previously published values. The results presented here represent the largest and most comprehensive analysis to date on the effects of compression on optically derived tissue properties. Mammographic related compression alters the oxygen saturation of hemoglobin in the breast. Additionally, differences in breast density can be ascertained using the NIRST/DBT platform by measuring the optically derived tissue properties of water, lipids, and hemoglobin. This outcome is critical for the development and success of the NIRST/DBT platform, which benefits from the synergy of its two component technologies. From the mammography perspective, NIRST contributes important physiological information. From the optical imaging perspective, the addition of DBT may improve the accuracy and resolution of the recovered optically derived property data as has been previously demonstrated for MRI. ${ }^{11}$ From a practical standpoint, the lower cost of the NIRST system design and the rapidity of the NIRST acquisition further adds to its clinical application. NIRST/DBT is a robust imaging technique that uses the detailed anatomic information from DBT to provide additional physiologic information that is highly correlated with breast density and minimally altered by changes in breast position or compression.

\section{Acknowledgments}

This work was funded by the National Institutes of Health Grant Nos. R01CA139449 and F30CA168079 and Radiological Society of North America RMS 1417.

\section{References}

1. S. Shapiro, P. Strax, and L. Venet, "Periodic breast cancer screening in reducing mortality from breast cancer," JAMA 215(11), 1777-1785 (1971).

2. L. Tabar et al., "Efficacy of breast cancer screening by age: new results from the Swedish two-county trial," Cancer 75(10), 2507-2517 (1995).

3. J. G. Elmore et al., "Ten-year risk of false positive screening mammograms and clinical breast examinations," N. Engl. J. Med. 338(16), 1089-1096 (1998).

4. Anon, "Final evidence summary: breast cancer: screening-US preventive services task force," http://www.uspreventiveservicestaskforce.org/ Page/SupportingDoc/breast-cancer-screening/final-evidence-summary9 (3 August 2015).

5. A. Bleyer and H. G. Welch, "Effect of three decades of screening mammography on breast-cancer incidence," N. Engl. J. Med. 367(21), 19982005 (2012).

6. C. Harding et al., "Breast cancer screening, incidence, and mortality across US counties," JAMA Intern. Med. 175(9), 1483-1489 (2015).

7. E. A. Rafferty et al., "Assessing radiologist performance using combined digital mammography and breast tomosynthesis compared with 
digital mammography alone: results of a multicenter, multireader trial," Radiology 266(1), 104-113 (2013).

8. P. Skaane et al., "Comparison of digital mammography alone and digital mammography plus tomosynthesis in a population-based screening program," Radiology 267(1), 47-56 (2013).

9. B. W. Pogue et al., "Image analysis methods for diffuse optical tomography," J. Biomed. Opt. 11(3), 033001 (2006).

10. Q. Fang et al., "Combined optical and X-ray tomosynthesis breast imaging," Radiology 258, 89-97 (2011).

11. B. Brooksby et al., "Imaging breast adipose and fibroglandular tissue molecular signatures by using hybrid MRI-guided near-infrared spectral tomography," Proc. Natl. Acad. Sci. U. S. A. 103, 8828-8833 (2006).

12. V. Krishnaswamy et al., "A digital x-ray tomosynthesis coupled near infrared spectral tomography system for dual-modality breast imaging," Opt. Express 20(17), 19125-19136 (2012).

13. S. A. Carp et al., "Hemodynamic signature of breast cancer under fractional mammographic compression using a dynamic diffuse optical tomography system," Biomed. Opt. Express 4(12), 2911 (2013).

14. S. P. Poplack et al., "Digital breast tomosynthesis: initial experience in 98 women with abnormal digital screening mammography," AJR Am.J. Roentgenol. 189(3), 616-623 (2007).

15. K. Michaelsen et al., "Characterization of materials for optimal nearinfrared and x-ray imaging of the breast," Biomed. Opt. Express 3(9), 2078 (2012).

16. A. E. Cerussi et al., "Diffuse optical spectroscopic imaging correlates with final pathological response in breast cancer neoadjuvant chemotherapy," Philos. Trans. Math. Phys. Eng. Sci. 369, 4512-4530 (2011).

17. S. Vedantham et al., "Semi-automated segmentation and classification of digital breast tomosynthesis reconstructed images," Conf. Proc. IEEE Eng. Med. Biol. Soc. 2011, 6188-6191 (2011).

18. S. Vedantham et al., "Digital breast tomosynthesis guided near infrared spectroscopy: volumetric estimates of fibroglandular fraction and breast density from tomosynthesis reconstructions," Biomed. Phys. Eng. Express 1(4), 045202 (2015).

19. B. W. Pogue et al., "Implicit and explicit prior information in near-infrared spectral imaging: accuracy, quantification and diagnostic value," Philos. Trans. Math. Phys. Eng. Sci. 369, 4531-4557 (2011).

20. M. Jermyn et al., "Fast segmentation and high-quality three-dimensional volume mesh creation from medical images for diffuse optical tomography," J. Biomed. Opt. 18(8), 086007 (2013).

21. Anon, "Advance data from vital and health statistics, number 347," http:// loristalter.com/wp-content/uploads/CDC_1960_2002.pdf (1 April 2014).

22. M. A. Helvie et al., "Breast thickness in routine mammograms: effect on image quality and radiation dose," Am. J. Roentgenol. 163(6), 13711374 (1994).

23. R. E. Hendrick et al., "Comparison of acquisition parameters and breast dose in digital mammography and screen-film mammography in the American College of Radiology Imaging Network digital mammographic imaging screening trial," AJR Am. J. Roentgenol. 194(2), 362-369 (2010).

24. M. J. Yaffe et al., "The myth of the 50-50 breast," Med. Phys. 36(12), 5437-5443 (2009).

25. S. Vedantham et al., "Dedicated breast CT: fibroglandular volume measurements in a diagnostic population," Med. Phys. 39(12), 7317-7328 (2012).

26. K. M. Blackmore et al., "The association between breast tissue optical content and mammographic density in pre- and post-menopausal women," PLoS One 10(1), 1-16 (2015).

27. X. Intes, "Time-domain optical mammography SoftScan: initial results," Acad. Radiol. 12(8), 934-947 (2005).

28. S. Srinivasan et al., "In vivo hemoglobin and water concentrations, oxygen saturation, and scattering estimates from near-infrared breast tomography using spectral reconstruction1," Acad. Radiol. 13, 195202 (2006).

29. P. Taroni et al., "Breast tissue composition and its dependence on demographic risk factors for breast cancer: non-invasive assessment by time domain diffuse optical spectroscopy," PLoS One 10(6), 1-16 (2015).

30. D. Grosenick et al., "Time-domain scanning optical mammography: II. Optical properties and tissue parameters of 87 carcinomas," Phys. Med. Biol. 50, 2451-2468 (2005).
31. Q. Fang et al., "Combined optical imaging and mammography of the healthy breast: optical contrast derived from breast structure and compression," IEEE Trans. Med. Imaging 28(1), 30-42 (2009).

32. A. Cerussi et al., "In vivo absorption, scattering, and physiologic properties of 58 malignant breast tumors determined by broadband diffuse optical spectroscopy," J. Biomed. Opt. 11, 044005 (2006).

33. V. Ntziachristos et al., "MRI-guided diffuse optical spectroscopy of malignant and benign breast lesions," Neoplasia 4(4), 347-354 (2002).

34. L. Spinelli et al., "Characterization of female breast lesions from multiwavelength time-resolved optical mammography," Phys. Med. Biol. 50(11), 2489-2502 (2005).

35. J. Wang et al., "Near-infrared tomography of breast cancer hemoglobin, water, lipid, and scattering using combined frequency domain and $\mathrm{cw}$ measurement," Opt. Lett. 35(1), 82-84 (2010).

36. B. J. Tromberg et al., "Imaging in breast cancer: diffuse optics in breast cancer: detecting tumors in pre-menopausal women and monitoring neoadjuvant chemotherapy," Breast Cancer Res. 7(6), 279 (2005).

37. W. Mo et al., "Quantitative characterization of optical and physiological parameters in normal breasts using time-resolved spectroscopy: in vivo results of 19 Singapore women," J. Biomed. Opt. 14(6), 064004 (2009).

38. S. Ueda et al., "Optical imaging of tumor vascularity associated with proliferation and glucose metabolism in early breast cancer: clinical application of total hemoglobin measurements in the breast," $B M C$ Cancer 13(1), 514 (2013).

39. Q. Zhu et al., "Early-stage invasive breast cancers: potential role of optical tomography with US localization in assisting diagnosis," Radiology 256(2), 367-378 (2010).

40. D. R. Busch et al., "Blood flow reduction in breast tissue due to mammographic compression," Acad. Radiol. 21(2), 151-161 (2014).

41. S. A. Carp et al., "Compression-induced changes in the physiological state of the breast as observed through frequency domain photon migration measurements," J. Biomed. Opt. 11(6), 064016 (2006).

42. S. A. Carp et al., "Dynamic functional and mechanical response of breast tissue to compression," Opt. Express 16, 16064-16078 (2008).

Kelly E. Michaelsen recently graduated with an $\mathrm{MD}$ and $\mathrm{PhD}$ degrees from Dartmouth Medical School. Her PhD was in biomedical engineering at Thayer School of Engineering. She graduated from Dartmouth College with honors, majoring in physics, and minoring in chemistry while pursuing research in particle physics. She is interested in developing imaging methods that translate into more effective screening, diagnosis, and treatment of disease. Her current research focuses on combining $x$-ray and optical imaging modalities for breast cancer surveillance.

Venkataramanan Krishnaswamy received his $\mathrm{PhD}$ in optical science and engineering from the University of Alabama in Huntsville with specialization in optical systems design and engineering. $\mathrm{He}$ is currently a research faculty member at Thayer School of Engineering, Dartmouth College. His current areas of research include imaging localized tissue scatter response using structured light and dark-field confocal spectroscopy approaches and multimodal imaging systems development combining near-infrared spectral tomography with mainstream clinical imaging modalities.

Linxi Shi is a PhD candidate in Nuclear and Radiological Engineering and Medical Physics Programs at Georgia Institute of Technology. Her research is mainly in developing imaging technologies of advanced $\mathrm{x}$-ray systems and its translations into clinical application. Current research has been focused on quantitative improvement of cone beam CT for its applications in breast cancer diagnosis and more efficient radiation therapy delivery.

Srinivasan Vedantham is an associate professor of radiology at the University of Massachusetts Medical School. His research is focused on the development and clinical translation of advanced x-ray imaging systems and techniques. His research is funded by the National Institutes of Health and he is actively conducting research on translating breast computed tomography to the clinic. He has published more than 90 articles and is a fellow of the American Association of Physicists in Medicine.

Andrew Karellas received his $\mathrm{PhD}$ in medical physics from the University of California, Los Angeles. He is a professor of radiology 
and the director of radiological physics at the University of Massachusetts Medical School. His current research interests are on new x-ray tomographic imaging techniques of the breast and on the development and evaluation of $x$-ray imaging detectors. His research has been funded over the years by the National Institutes of Health.

Brian W. Pogue is a professor of engineering, physics, and astronomy at Dartmouth College, and adjunct professor of surgery at the Geisel School of Medicine. His research is in optical imaging systems, with a focus on molecular and structural imaging of cancer for surgical guidance and imaging radiation therapy. He has published 240 peer-reviewed papers. His research is funded by the National Cancer Institute, and he is a fellow of the Optical Society of America.

Keith D. Paulsen is currently the Robert A. Pritzker professor of biomedical engineering at Thayer School of Engineering at Dartmouth, professor of radiology at the Geisel School of Medicine, and director of the Advanced Imaging Center at Dartmouth Hitchcock Medical Center. His research has focused on the development and translation of advanced imaging technology, primarily for cancer detection, diagnosis, therapy monitoring, and surgical guidance. He has authored more than 350 archival publications with an active research program, continuously funded by the National Institutes of Health for 25 years.

Steven P. Poplack is a clinical breast radiologist and associate professor of radiology at the Washington University School of Medicine in St. Louis. His research focuses on novel breast imaging and breast intervention technology development. $\mathrm{He}$ is recognized internationally as an educator and has 65 peer reviewed publications. He serves as director at large for the Society of Breast Imaging and is a fellow of the Society of Breast Imaging and American College of Radiology. 